\title{
Characterization of polynomial decay rate for the solution of linear evolution equation
}

\author{
Zhuangyi Liu * Bopeng Rao ${ }^{\dagger}$
}

\begin{abstract}
In this paper, we study the decay rate of solutions to strongly stable, but not exponentially stable linear evolution equations. It is known that the resolvent operator of such an equation must be unbounded on the imaginary axis. Our main result is an estimate of the decay rate when the unboundedness is of polynomial order. We then apply our main theorem to three strongly stable but not exponentially stable systems to obtain the decay rate, which is not available in the literature.
\end{abstract}

\section{Introduction}

We consider a linear evolution equation on Hilbert space $\mathcal{H}$ :

$$
\left\{\begin{array}{l}
\frac{d x}{d t}=\mathcal{A} x \\
x(0)=x_{0} .
\end{array}\right.
$$

Assume that

(H1). $\mathcal{A}$ generates a bounded $C_{0}$ semigroup $S(t)=e^{\mathcal{A} t}$ on $\mathcal{H}$.

(H2). $i \mathbb{R} \cap \sigma(\mathcal{A})=\emptyset$.

*Department of Mathematics and Statistics, University of Minnesota, Duluth, MN 55812-2496, USA

†Institut de Recherche Mathématique Avancée, Université de Loius Pasteur de Strasbourg, 7 Rue RenéDescartes, 67084 Strasbourg Cedex, France. 
The solution to $(1.1)$ is

$$
x(t)=S(t) x_{0} .
$$

We say that (1.1) is strongly stable if

$$
\lim _{t \rightarrow \infty}\|x(t)\|_{\mathcal{H}}=0
$$

for all $x_{0} \in \mathcal{H}$; is exponentially stable if there exist constants $M, \alpha>0$ such that

$$
\|x(t)\|_{\mathcal{H}} \leq M e^{-\alpha t}\left\|x_{0}\right\|_{\mathcal{H}}, \quad t>0
$$

for all $x_{0} \in \mathcal{H}$. There are many systems which are strongly stable, but not exponentially stable. For example, when damping in an elastic system is located only on a subdomain or on part of the boundary, its energy still dissipates, but may not at a uniform rate. In that case, other kind of decay rates had been introduced. If there exists a positive function $f(t)$ with $\lim _{t \rightarrow \infty} f(t)=0$ such that

$$
\|x(t)\|_{\mathcal{H}} \leq f(t)\left\|x_{0}\right\|_{\mathcal{D}(\mathcal{A})}, \quad t>0
$$

we say the solution of $(1.1)$ decays at a rate of $f(t)$ for all $x_{0} \in \mathcal{D}(\mathcal{A})$. Note that the norm on the right-hand side of $(1.4)$ can not be the $\mathcal{H}$-norm. Otherwise, by the semigroup properties, (1.4) implies (1.3).

Several time domain methods for the polynomial decay rate estimate exist in the literature. Energy estimate method combined with multiplier technique can be found in $[R],[R R]$, [RW]. An energy inequality was established in $[\mathrm{Ru}]$ as sufficient condition for polynomial decay rate $1 / t$. This inequality was generalized in [JTZ]. A Riesz basis method was used in $[\mathrm{LbLw}]$ which gives the polynomial decay rate based on the asymptotic relation of the real and imaginary part of the eigenvalues. Another method by using Ingham's inequality can be found in $[\mathrm{ALT}]$. It is not our intention to do a complete review on this subject here. We refer the readers to the references in the mentioned above for more information.

The semigroup $S(t)$ is strongly stable if condition (H2) holds (see [H1],[H3]); is exponentially stable if and only if (H2) and

$$
\sup \left\{\left\|(i \beta-\mathcal{A})^{-1}\right\|_{\mathcal{H}} \mid \beta \in \mathbb{R}\right\}<\infty
$$


are satisfied (see $[\mathrm{H} 2],[\mathrm{Pr}])$. Moreover, $S(t)$ is analytic (see $[\mathrm{LY}],[\mathrm{LZ}]$ ) if

$$
\left\|(i \beta-\mathcal{A})^{-1}\right\|_{\mathcal{H}}=O\left(\frac{1}{\beta}\right), \quad \beta \rightarrow \infty .
$$

These frequency domain results have been used to study the asymptotic behavior and smoothness of the solution to a given linear evolution equation.

One can observe that the growth rate of the resolvent operator on the imaginary axis is related to the decay rate of the solution to (1.1). If we know that (1.1) is strongly stable but not exponentially stable, then condition (1.5) must fail, i.e., the resolvent operator is unbounded on the imaginary axis. In his study on wave equation with internal or boundary damping, Lebeau [L] had used this approach to get decay rate estimate. Our main goal is to provide a characterization of the decay rate of solution to (1.1) by the order of unboundedness of the resolvent operator on the imaginary axis, particularly for the case of $\left\|(i \beta-\mathcal{A})^{-1}\right\|_{\mathcal{H}}=$ $O\left(\beta^{l}\right)$.

This paper is organized as following: section 2 is devoted to the statement and proof of the main theorem. In section 3, we apply our theorem to three strongly stable but not exponentially stable systems. The first one is an elastic string with Kelvin-Voigt damping on a segment of the string and damping coefficient discontinuous at the interface. The second one is a weakly coupled n-dimensional wave equation and heat equation. The last one is a wave equation on a square domain with viscous damping on a subdomain which contains a vertical strip. We are able to estimate the decay rates for these systems, which are not available in the literature.

\section{Main Theorem}

We further assume that

(H3). $\sup _{|\beta| \geq 1} \frac{1}{\beta^{l}}\left\|(\boldsymbol{i} \beta-\mathcal{A})^{-1}\right\| \leq M$ for some $l>0$.

By the resolvent identity

$$
\begin{aligned}
(-\alpha+\boldsymbol{i} \beta-\mathcal{A})^{-1} & =(\boldsymbol{i} \beta-\mathcal{A})^{-1}\left[I-\alpha(\boldsymbol{i} \beta-\mathcal{A})^{-1}\right]^{-1} \\
& =(\boldsymbol{i} \beta-\mathcal{A})^{-1}\left[I-\alpha|\beta|^{l} \frac{(\boldsymbol{i} \beta-\mathcal{A})^{-1}}{|\beta|^{l}}\right]^{-1}
\end{aligned}
$$


we see that $-\alpha+\boldsymbol{i} \beta-\mathcal{A}$ is invertible when $\left|\alpha \beta^{l}\right| \leq \frac{1}{2 M}$ and $|\beta| \geq 1$. Moreover, $\sup _{|\beta| \geq 1} \frac{1}{\beta^{l}} \|(-\alpha+$ $\boldsymbol{i} \beta-\mathcal{A})^{-1} \| \leq 2 M$. Since $\mathcal{A}^{-1}$ exists, from the continuity of the resolvent operator, there is an constant $\varepsilon>0$ and a curve

$$
\Gamma^{\varepsilon}=\left\{z=-\frac{\varepsilon}{\eta^{l}}+\boldsymbol{i} \eta|| \eta \mid \geq 1\right\} \cup\{z=-\varepsilon+\boldsymbol{i} \eta|| \eta \mid<1\}
$$

such that the region on the right-hand side of $\Gamma^{\varepsilon}$ (including the boundary) belongs to the resolvent set of $\mathcal{A}$.

Lemma 2.1 The following identity holds for all integer $k \geq 1$ and all $x \in \mathcal{H}$ :

$$
e^{t \mathcal{A}}(I-\mathcal{A})^{-k} x=\frac{1}{2 \pi \boldsymbol{i}} \int_{\Gamma} \frac{(\zeta I-\mathcal{A})^{-1} x}{(1-\zeta)^{k}} e^{t \zeta} d \zeta=\frac{1}{2 \pi \boldsymbol{i}} \int_{\Gamma^{\varepsilon}} \frac{(\zeta I-\mathcal{A})^{-1} x}{(1-\zeta)^{k}} e^{t \zeta} d \zeta
$$

where $\Gamma=\left\{\zeta=\frac{1}{2}+\boldsymbol{i} \eta \mid \eta \in \mathbb{R}\right\}$.

Proof. Let $B=\mathcal{A}-I$. Then by equation (1.13) in $[\mathrm{Na}]$,

$$
e^{t B} B^{-k} x=\frac{1}{2 \pi \boldsymbol{i}} \int_{-\frac{1}{2}-\boldsymbol{i}_{\infty}}^{-\frac{1}{2}+\boldsymbol{i}_{\infty}} \frac{(\lambda I-B)^{-1} x}{\lambda^{k}} e^{t \lambda} d \lambda
$$

for every $x \in \mathcal{H}$ and $t>0$. Returning to the original operator $\mathcal{A}$ and letting $\zeta=\lambda+1$, we obtain the first equality. Using the following estimates:

$$
\left\{\begin{array}{l}
\left\|(\zeta I-\mathcal{A})^{-1} x\right\| \leq C\|x\| \\
\frac{1}{|1-\zeta|} \leq \frac{2}{|\zeta|}
\end{array}\right.
$$

the second equality in (2.3) can be easily established by a contour integration.

Theorem 2.1 Suppose that the hypotheses (H1)-(H3) hold. Then, for any positive integer $k$ there exists a constant $C_{k}>0$ such that

$$
\left\|e^{t \mathcal{A}} z\right\|_{\mathcal{H}} \leq C_{k}\left(\frac{\ln t}{t}\right)^{\frac{k}{l}}(\ln t)\|x\|_{\mathcal{D}\left(\mathcal{A}^{k}\right)}
$$

for all $x \in \mathcal{D}\left(\mathcal{A}^{k}\right)$.

Remark: Estimate (2.6) is not optimal. In the papers $[R]$ and $[R W]$, they got the best decay rate $\frac{1}{t^{1 / 2}}$ for the solution of an hybrid system. But using (2.6), we can only get the decay rate $\frac{(\ln t)^{3 / 2}}{t^{1 / 2}}$. Our effort to remove the $\ln t$ term in $(2.6)$ has not been successful. 
Proof of Theorem 2.1. Inserting a weight function to the identities in (2.3), we have

$$
\begin{aligned}
e^{t \mathcal{A}}(I-\mathcal{A})^{-k} x & =\frac{1}{2 \pi \boldsymbol{i}} \int_{\Gamma} \int_{-\infty}^{+\infty} \frac{1}{\sqrt{2 \pi}} e^{-\frac{\left(\lambda+\boldsymbol{i}_{\zeta)^{2}}\right.}{2}} d \lambda \frac{(\zeta-\mathcal{A})^{-1} x}{(1-\zeta)^{k}} e^{t \zeta} d \zeta \\
& =I_{1}+I_{2}
\end{aligned}
$$

where

$$
I_{1}=\int_{\Gamma^{\varepsilon}} \int_{|\lambda| \leq c_{1}\left(\frac{t}{\ln t}\right)^{1 / l}}(\cdots) d \lambda d \zeta, \quad I_{2}=\int_{\Gamma} \int_{|\lambda|>c_{1}\left(\frac{t}{\ln t}\right)^{1 / l}}(\cdots) d \lambda d \zeta
$$

Estimation of $I_{1}$

For $\zeta \in \Gamma^{\varepsilon}$, we have

$$
\begin{aligned}
&(\lambda+\boldsymbol{i} \zeta)^{2}= \begin{cases}(\lambda-\eta)^{2}-\frac{\varepsilon^{2}}{\eta^{2 l}}-2(\lambda-\eta) \frac{\varepsilon}{\eta^{l}} \boldsymbol{i} & |\eta| \geq 1 \\
(\lambda-\eta)^{2}-\varepsilon^{2}-2(\lambda-\eta) \varepsilon \boldsymbol{i} & |\eta|<1\end{cases} \\
&|1-\zeta| \geq \begin{cases}C(1+|\eta|) & |\eta| \geq 1 \\
C & |\eta|<1,\end{cases} \\
&\left\|(\zeta-\mathcal{A})^{-1} x\right\| \leq \begin{cases}\frac{C}{\varepsilon}|\eta|^{l}\|x\| & |\eta| \geq 1 \\
\frac{C}{\varepsilon}\|x\| & |\eta|<1 .\end{cases}
\end{aligned}
$$

Break $I_{1}$ as follows

$$
\begin{aligned}
I_{1} & =\int_{|\lambda| \leq \alpha_{1}} \int_{|\eta|<1}(\cdots) d \zeta d \lambda+\int_{|\lambda| \leq \alpha_{1}} \int_{\alpha_{2} \geq|\eta| \geq 1}(\cdots) d \zeta d \lambda+\int_{|\lambda| \leq \alpha_{1}} \int_{|\eta|>\alpha_{2}}(\cdots) d \zeta d \lambda \\
& =I_{1,1}+I_{1,2}+I_{1,3}
\end{aligned}
$$

where $\alpha_{i}=\left(c_{i} \frac{t}{\ln t}\right)^{\frac{1}{l}}$ and $c_{i}>0$ to be chosen. Then

$$
\left|I_{1,1}\right| \leq C \int_{|\lambda| \leq \alpha_{1}} \int_{|\eta|<1} e^{-\varepsilon t} d \eta d \lambda\|x\| \leq 2 C \alpha_{1} e^{-\varepsilon t}\|x\|=O\left(t^{-\infty}\right)\|x\|,
$$

and

$$
\begin{aligned}
\left|I_{1,2}\right| & \leq C \int_{|\lambda| \leq \alpha_{1}} \int_{\alpha_{2} \geq|\eta| \geq 1} e^{-\frac{\varepsilon t}{|\eta|^{+}}+\frac{\varepsilon^{2}}{2|\eta|^{2 l}}} \frac{|\eta|^{l}}{(1+|\eta|)^{k}} d \eta d \lambda\|x\| \\
& \leq C \alpha_{1} \alpha_{2} e^{-\frac{\varepsilon t}{2 \alpha_{2}^{l}}\left(\alpha_{2}\right)^{l-k}\|x\|} \\
& =C\left(\frac{t}{\ln t}\right)^{\frac{2+l-k}{l}} t^{-\frac{\varepsilon}{c_{2}}}\|x\|=O\left(t^{-\infty}\right)\|x\|
\end{aligned}
$$

provided that $c_{2}$ is small enough. Once $c_{2}$ is fixed, we choose $c_{1} \in\left(0, c_{2}\right)$ such that

$$
(\lambda-\eta)^{2} \geq 2 \delta\left(\eta^{2}+\lambda^{2}\right) \geq 2 \delta \eta^{2}
$$


for some $\delta>0$ when $|\lambda| \leq \alpha_{1}$ and $|\eta| \geq \alpha_{2}$. Therefore,

$$
\begin{aligned}
\left|I_{1,3}\right| & \leq \int_{|\lambda| \leq \alpha_{1}} \int_{|\eta|>\alpha_{2}} \frac{|\eta|^{l}}{(1+|\eta|)^{k}} e^{-\frac{1}{2}\left[(\lambda-\eta)^{2}+\frac{2 \varepsilon t}{|\eta|^{l}}-\frac{\varepsilon^{2}}{\left.\eta^{2 l}\right]}\right.} d \eta d \lambda\|x\| \\
& \leq C \alpha_{1} \int_{|\eta|>\alpha_{2}} \frac{|\eta|^{l}}{(1+|\eta|)^{k}} e^{-\delta \eta^{2}} d \eta\|x\|=O\left(t^{-\infty}\right)\|x\|
\end{aligned}
$$

Combining (2.9)-(2.11), we have

$$
\left|I_{1}\right| \leq O\left(t^{-\infty}\right)\|x\|
$$

Estimation of $I_{2}$

We rewrite $I_{2}$ as

$$
I_{2}=e^{t \mathcal{A}} J
$$

where

$$
J=\frac{1}{2 \pi \boldsymbol{i}} \int_{\Gamma} \int_{|\lambda|>\alpha_{1}} \frac{1}{\sqrt{2 \pi}} e^{-\frac{\left(\lambda+\boldsymbol{i}_{\zeta)^{2}}\right.}{2}} d \lambda \frac{(\zeta-\mathcal{A})^{-1} x}{(1-\zeta)^{k}} d \zeta
$$

Define the set on complex $\lambda$ plane

$$
\Omega_{t}=\left\{\lambda=\alpha_{1}-\frac{\boldsymbol{i}}{2}+\mu e^{-\boldsymbol{i} \theta} \mid \mu \geq 0, \theta \in\left[0, \frac{\pi}{8}\right]\right\} .
$$

For any $\lambda \in \Omega_{t}$, we construct a contour on $\zeta$ plane

$$
\begin{aligned}
D_{\lambda}^{+} & =\{\zeta=\boldsymbol{i}(1+\eta)-\operatorname{Im} \lambda \mid \eta \geq 0\} \\
D_{\lambda}^{-} & =\left\{\zeta=\boldsymbol{i}(1+\eta)+\frac{1}{2} \mid \eta \leq 0\right\} \\
D_{\lambda}^{0} & \left.=\left\{\zeta=\boldsymbol{i}+\frac{1}{2}+\eta\right) \mid 0 \leq \eta \leq-\operatorname{Im} \lambda-\frac{1}{2}\right\}
\end{aligned}
$$

Let

$$
H_{\lambda}(x, \zeta)=\frac{1}{2 \pi \boldsymbol{i}} \frac{1}{\sqrt{2 \pi}} \frac{(\zeta-\mathcal{A})^{-1} x}{(1-\zeta)^{k}} e^{-\frac{1}{2}(\lambda+\boldsymbol{i} \zeta)^{2}}, \quad \lambda \in \Omega_{t}
$$

Then $J=J^{+}+J^{-}$where

$$
J^{ \pm}=\int_{ \pm \lambda \geq \alpha_{1}} \int_{\Gamma} H_{\lambda}(x, \zeta) d \zeta d \lambda=\int_{ \pm \alpha-\frac{i}{2}}^{ \pm \infty-\frac{i}{2}} \int_{\Gamma} H_{\lambda}(x, \zeta) d \zeta d \lambda=\int_{ \pm \alpha_{1}-\frac{i}{2}}^{ \pm \infty-\frac{i}{2}} \int_{D_{\lambda}^{-} \cup D_{\lambda}^{0} \cup D_{\lambda}^{+}} H_{\lambda}(x, \zeta) d \zeta d \lambda
$$

Note that the contour of integration for $\lambda$ and $\zeta$ have been shifted. In what follows, we will carry out the estimation of $J^{+} . J^{-}$can be estimated similarly. 
For $\lambda \in \Omega_{t}$ and $\zeta \in D_{\lambda}^{0}$,

$$
\lambda+\boldsymbol{i} \zeta=\left(\alpha_{1}+\mu \cos \theta-1\right)+\boldsymbol{i}(\eta-\mu \sin \theta), \quad \eta \leq \mu \sin \theta
$$

Then

$$
R e(\lambda+\boldsymbol{i} \zeta)^{2}=\left(\alpha_{1}+\mu \cos \theta-1\right)^{2}-(-\eta+\mu \sin \theta)^{2} \geq 2 \delta\left(\mu^{2}+\eta^{2}+\alpha_{1}^{2}\right) .
$$

For $\lambda \in \Omega_{t}$ and $\zeta \in D_{\lambda}^{-}$,

$$
\lambda+\boldsymbol{i} \zeta=\left(\alpha_{1}+\mu \cos \theta-\eta-1\right)+\boldsymbol{i} \mu \sin \theta, \quad \eta \leq 0 .
$$

Then

$$
\begin{aligned}
\operatorname{Re}(\lambda+\boldsymbol{i} \zeta)^{2} & =\left(\alpha_{1}+\mu \cos \theta-\eta-1\right)^{2}-\mu^{2} \sin ^{2} \theta \\
& \geq \mu^{2} \cos 2 \theta+\eta^{2}+\left(\alpha_{1}-1\right)^{2} \\
& \geq 2 \delta\left(\mu^{2}+\eta^{2}+\alpha_{1}^{2}\right)
\end{aligned}
$$

It follows that

$$
\left|\int_{D_{\lambda}^{-} \cup D_{\lambda}^{0}} H_{\lambda}(x, \zeta) d \zeta\right| \leq C\|x\| e^{-\delta \alpha_{1}^{2}} \int_{0}^{+\infty} \frac{e^{-\delta\left(\mu^{2}+\eta^{2}\right)}}{(1+|\eta|)^{k}} d \eta \leq C\|x\| e^{-\delta \alpha_{1}^{2}} e^{-\delta \mu^{2}} .
$$

For $\lambda \in \Omega_{t}$ and $\zeta \in D_{\lambda}^{+}$, we have

$$
\begin{aligned}
(\lambda+\boldsymbol{i} \zeta)^{2} & =\left(\alpha_{1}+\mu \cos \theta-\eta-1\right)^{2} \\
\left\|(\zeta-\mathcal{A})^{-1}\right\| & \leq \frac{C}{|\operatorname{Re} \zeta|}=\frac{C}{|\operatorname{Im} \lambda|} \leq \frac{C}{\mu \sin \theta+1} \\
\left\|(\zeta-\mathcal{A})^{-1} x\right\| & \leq \frac{C_{x}}{1+|\zeta|} \leq \frac{C_{x}}{1+|\eta|}
\end{aligned}
$$

Then, it follows from (2.16) and (2.18) that

$$
\begin{aligned}
\left|\int_{D_{\lambda}^{+}} H_{\lambda}(x, \zeta) d \zeta\right| & \leq C_{x} \int_{0}^{+\infty} \frac{e^{-\frac{1}{2}\left[\left(\alpha_{1}+\mu \cos \theta-1\right)-\eta\right]^{2}}}{(1+|\eta|)^{k+1}} d \eta \\
& \leq \frac{C_{x}}{\left(\alpha_{1}+\mu \cos \theta\right)^{k+1}}
\end{aligned}
$$

Therefore, by contour integration, we can rewrite

$$
J^{+}=\int_{\gamma} \int_{D_{\lambda}^{-} \cup D_{\lambda}^{0} \cup D_{\lambda}^{+}} H_{\lambda}(x, \zeta) d \zeta d \lambda
$$


where $\gamma=\left\{\lambda=\alpha_{1}-\frac{i}{2}+\mu e^{-i \frac{\pi}{8}} \mid \mu \geq 0\right\}$. On the other hand, using (2.16)-(2.17), we have

$$
\begin{aligned}
\left|\int_{D_{\lambda}^{+}} H_{\lambda}(x, \zeta) d \zeta\right| & \leq \frac{C\|x\|}{\left|\mu \sin \frac{\pi}{8}+1\right|} \int_{0}^{+\infty} \frac{e^{-\frac{1}{2}\left[\left(\alpha_{1}+\mu \cos \frac{\pi}{8}-1\right)-\eta\right]^{2}}}{(1+|\eta|)^{k}} d \eta \\
& \leq \frac{C\|x\|}{\left|\mu \sin \frac{\pi}{8}+1\right|} \cdot \frac{1}{\left(\alpha_{1}+\mu \cos \frac{\pi}{8}\right)^{k}} \\
& \leq \frac{C\|x\|}{(\mu+1)\left(\mu+\alpha_{1}\right)^{k}} .
\end{aligned}
$$

Combining (2.15) and (2.20), we conclude that

$$
\begin{aligned}
\left|J^{+}\right| & \leq C\|x\| \int_{0}^{+\infty}\left[e^{-\delta \alpha_{1}^{2}} e^{-\delta \mu^{2}}+\frac{1}{(\mu+1)\left(\mu+\alpha_{1}\right)^{k}}\right] d \mu \\
& \leq C\|x\|\left(e^{-\delta \alpha_{1}^{2}}+\frac{\ln \alpha_{1}}{\alpha_{1}^{k}}\right) \\
& \leq C\|x\| \ln \left(c_{1} \frac{t}{\ln t}\right)^{\frac{1}{l}}\left(c_{1} \frac{t}{\ln t}\right)^{-\frac{k}{l}} \\
& \leq C\|x\|\left(\frac{\ln t}{t}\right)^{\frac{k}{l}} \ln t .
\end{aligned}
$$

After a similar estimation of $J^{-}$, we obtain

$$
|J| \leq C\|x\|\left(\frac{\ln t}{t}\right)^{\frac{k}{l}} \ln t .
$$

Therefore,

$$
\left|I_{2}\right| \leq C\|x\|\left(\frac{\ln t}{t}\right)^{\frac{k}{l}} \ln t .
$$

Finally, we conclude (2.6) from (2.7), (2.12) and (2.23).

\section{Applications}

In this section, we consider three strongly stable but not exponentially stable systems. By Theorem 2.1, we are able to obtain the decay rates for the solutions to these systems.

Example 1. We consider a 1-d wave equation with local Kelvin-Voigt damping and discontinuous coefficient functions:

$$
\left\{\begin{array}{l}
\rho(x) u_{t t}(x, t)-\left[a(x) u^{\prime}(x, t)+d(x) u_{t}^{\prime}(x, t)\right]^{\prime}=0 \quad \text { in }(0, L) \times \mathbb{R}^{+} \\
u(0, t)=u(L, t)=0 \\
u(x, 0)=u_{0}(x), u_{t}(x, 0)=u_{1}(x)
\end{array}\right.
$$


where $\rho(x), a(x) \geq c_{0}>0$ and $d(x)=0$ on $(0, \alpha), d(x) \geq c_{0}$ on $(\alpha, L), \rho, a \in H^{1}(0, \alpha) \cup$ $H^{1}(\alpha, L)$ and $d \in C(\alpha, L)$. The prime denotes the derivatives with respect to $x$.

The energy of this system is defined by

$$
E(t)=\frac{1}{2} \int_{0}^{L}\left[\rho(x)\left|u_{t}\right|^{2}+a(x)\left|u^{\prime}\right|^{2}\right] d x .
$$

Let

$$
\begin{array}{rlrl}
H(b, c) & =L^{2}(b, c), & \|v\|_{H(b, c)}^{2} & =\int_{b}^{c} \rho(x)|v(x)|^{2} d x, \\
V(b, c) & =H_{0}^{1}(b, c), & \|v\|_{V(b, c)}^{2}=\int_{b}^{c} a(x)\left|v^{\prime}(x)\right|^{2} d x,
\end{array}
$$

and

$$
\mathcal{H}=V(0, L) \times H(0, L), \quad\|(u, v)\|_{\mathcal{H}}^{2}=\|u\|_{V(0, L)}^{2}+\|v\|_{H(0, L)}^{2} .
$$

We then define in $\mathcal{H}$ that

$$
D(\mathcal{A})=\left\{(u, v) \mid v \in V, a u^{\prime}+d v^{\prime} \in H^{1}(0, L)\right\}
$$

and

$$
\mathcal{A}(u, v)=\left(v, \frac{1}{\rho}\left(a u^{\prime}+d v^{\prime}\right)^{\prime}\right)
$$

Thus (3.1) can be rewritten as an abstract evolution equation on $\mathcal{H}$ :

$$
\frac{d}{d t}(u, v)=\mathcal{A}(u, v), \quad(u(0), v(0))=\left(u_{0}, u_{1}\right)
$$

It is known that (see [LL]) $\mathcal{A}$ satisfies the hypotheses (H1) and (H2). Moreover, $e^{t \mathcal{A}}$ is not exponentially stable.

In what follows, we show that the hypothesis (H3) holds here for $l=\frac{1}{2}$. If it is false, then there exist $\beta_{n} \in \mathbb{R}^{+},\left(u_{n}, v_{n}\right) \in D(\mathcal{A}), n=1,2, \cdots$, with

$$
\left\|\left(u_{n}, v_{n}\right)\right\|_{\mathcal{H}}=1, \quad \beta_{n} \rightarrow \infty
$$

and

$$
\beta_{n}^{\frac{1}{2}}\left(\boldsymbol{i} \beta_{n}-\mathcal{A}\right)\left(u_{n}, v_{n}\right) \equiv\left(f_{n}, g_{n}\right) \rightarrow 0 \text { in } \mathcal{H}
$$

i.e.,

$$
\begin{aligned}
\beta_{n}^{\frac{1}{2}}\left(\boldsymbol{i} \beta_{n} u_{n}-v_{n}\right) & =f_{n} \rightarrow 0 \quad \text { in } V(0, L), \\
\beta_{n}^{\frac{1}{2}}\left(\boldsymbol{i} \beta_{n} v_{n}-\frac{1}{\rho} T_{n}^{\prime}\right) & =g_{n} \rightarrow 0 \quad \text { in } H(0, L) .
\end{aligned}
$$


where $T_{n}=a u_{n}^{\prime}+d v_{n}^{\prime}$. Our goal is to find a contradiction to (3.6).

We first consider (3.8)-(3.9) on the interval $(\alpha, L)$. From $(3.7)$, we obtain

$$
\beta_{n}^{\frac{1}{2}} \int_{\alpha}^{L} d(x)\left|v_{n}^{\prime}\right|^{2} d x=\operatorname{Re}\left\langle\beta_{n}^{\frac{1}{2}}\left(\boldsymbol{i} \beta_{n}-\mathcal{A}\right)\left(u_{n}, v_{n}\right),\left(u_{n}, v_{n}\right)\right\rangle_{\mathcal{H}} \rightarrow 0
$$

which implies that

$$
\left\|\beta_{n}^{\frac{1}{4}} v_{n}^{\prime}\right\|_{H(\alpha, L)} \rightarrow 0, \quad\left\|\beta_{n}^{\frac{5}{4}} u_{n}^{\prime}\right\|_{H(\alpha, L)} \rightarrow 0
$$

Thus, we also have

$$
\left\|\beta_{n}^{\frac{1}{4}} T_{n}\right\|_{H(\alpha, L)} \rightarrow 0
$$

we take inner product of (3.9) with $v_{n}$ in $H(\alpha, L)$ to obtain

$$
\boldsymbol{i}\left\|\beta_{n}^{\frac{3}{4}} v_{n}\right\|_{H(\alpha, L)}^{2}+\beta_{n}^{\frac{1}{2}} T_{n}\left(\alpha^{+}\right) \bar{v}_{n}\left(\alpha^{+}\right)+\left\langle\beta_{n}^{\frac{1}{4}} T_{n}, \beta_{n}^{\frac{1}{4}} v_{n}^{\prime}\right\rangle_{L^{2}(\alpha, L)} \rightarrow 0 .
$$

The third term converges to zero due to (3.11) and (3.12). For the second term, we have the following estimate:

$$
\begin{aligned}
\beta_{n}^{\frac{1}{2}}\left|T_{n}\left(\alpha^{+}\right) \| v_{n}\left(\alpha^{+}\right)\right| & \leq C \beta_{n}^{\frac{1}{2}}\left\|v_{n}\right\|_{H(\alpha, L)}^{\frac{1}{2}}\left\|v_{n}^{\prime}\right\|_{H(\alpha, L)}^{\frac{1}{2}}\left\|T_{n}\right\|_{H(\alpha, L)}^{\frac{1}{2}}\left\|T_{n}^{\prime}\right\|_{H(\alpha, L)}^{\frac{1}{2}} \\
& =C\left\|\beta_{n}^{\frac{1}{4}} v_{n}^{\prime}\right\|_{H(\alpha, L)}^{\frac{1}{2}}\left\|\beta_{n}^{\frac{1}{4}} T_{n}\right\|_{H(\alpha, L)}^{\frac{1}{2}}\left\|\beta_{n}^{\frac{3}{4}} v_{n}\right\|_{H(\alpha, L)}+o(1) \\
& =o(1)\left(1+\left\|\beta_{n}^{\frac{3}{4}} v_{n}\right\|_{H(\alpha, L)}\right)
\end{aligned}
$$

where we have used $\left\|\beta_{n} v_{n}\right\|_{H(\alpha, L)} \sim\left\|T_{n}^{\prime}\right\|_{H(\alpha, L)}$ from (3.9). Now (3.13) leads to

$$
\left\|\beta_{n}^{\frac{3}{4}} v_{n}\right\|_{H(\alpha, L)} \rightarrow 0
$$

Next, we divide (3.9) by $\beta_{n}^{\frac{1}{2}}$, then take inner product with $(L-x) T_{n}$ in $H(\alpha, L)$ to get

$$
\operatorname{Re}\left\langle\beta_{n}^{\frac{3}{4}} v_{n},(L-x) \beta_{n}^{\frac{1}{4}} T_{n}\right\rangle_{H(\alpha, L)}-\frac{1}{2}(L-\alpha)\left|T_{n}\left(\alpha^{+}\right)\right|^{2}+\frac{1}{2}\left\|T_{n}\right\|_{L^{2}(\alpha, L)}^{2} \rightarrow 0 .
$$

Since the first the third terms converge to zero, we see that

$$
\left|T_{n}\left(\alpha^{+}\right)\right| \rightarrow 0
$$

On the other hand, by the Trace theorem and (3.11),

$$
\left|\beta_{n} u_{n}\left(\alpha^{+}\right)\right| \rightarrow 0
$$


Using the continuity conditions at $x=\alpha$, we arrive at

$$
\left\{\begin{array}{l}
\left|\beta_{n} u_{n}\left(\alpha^{-}\right)\right| \rightarrow 0 \\
\left|a\left(\alpha^{-}\right) u_{n}^{\prime}\left(\alpha^{-}\right)\right| \rightarrow 0
\end{array}\right.
$$

Now, we consider $(3.8)-(3.9)$ on the interval $(0, \alpha)$. Eliminating $v_{n}$ in (3.9) by (3.8) gives

$$
-\beta_{n}^{2} u_{n}-u_{n}^{\prime \prime}=\beta_{n}^{-\frac{1}{2}} g_{n}+\boldsymbol{i} \beta_{n}^{\frac{1}{2}} f_{n}
$$

Take the inner product of (3.18) with $q(x) u_{n}^{\prime}$ in $H(0, \alpha)$ where $q(0)=0$ and $q \in H^{1}(0, \alpha)$. A straight forward calculation shows that the real part of this inner product leads to the following

$\int_{0}^{\alpha}\left[\left(q^{\prime} \rho+q \rho^{\prime}\right)\left|\beta_{n} u_{n}\right|^{2}+\left(q^{\prime} a-q a^{\prime}\right)\left|u_{n}^{\prime}\right|^{2}\right] d x=q(\alpha) \rho\left(\alpha^{-}\right)\left|\beta_{n} u_{n}\left(\alpha^{-}\right)\right|^{2}+q(\alpha) a\left(\alpha^{-}\right)\left|u_{n}^{\prime}\left(\alpha^{-}\right)\right|^{2}+o(1)$.

The boundary terms on the right-hand side of (3.19) converge to zero which we have obtained in (3.17). Moreover, we can let

$$
q(x)=\int_{0}^{x} e^{\int_{x}^{s}\left(\left|\frac{\rho^{\prime}}{\rho}\right|+\left|\frac{a^{\prime}}{a}\right|\right) d \tau} d s
$$

and verify

$$
q^{\prime} \rho+q \rho^{\prime} \geq \rho, \quad q^{\prime} a-q a^{\prime} \geq a .
$$

It follows from (3.19) and (3.8) that

$$
\left\|v_{n}\right\|_{H(0, \alpha)} \rightarrow 0, \quad\left\|u_{n}\right\|_{V(0, \alpha)} \rightarrow 0
$$

Finally, we combine (3.20), (3.11) and (3.14) to obtain the promised contradiction. Thus, we have verified the hypothesis (H3).

Example 2. Consider a weakly coupled wave equation and heat equation on a bounded domain $\Omega \subset \mathbb{R}^{n}$

$$
\left\{\begin{array}{l}
u_{t t}(x, t)=\Delta u(x, t)-\gamma \theta(x, t) \\
\theta_{t}(x, t)=\gamma u_{t}(x, t)+k \Delta \theta(x, t) \\
\left.u\right|_{\partial \Omega}=\left.\theta\right|_{\partial \Omega}=0 \\
u(x, 0)=u_{0}(x), u_{t}(x, 0)=u_{1}(x), \theta(x, 0)=\theta_{0}(x)
\end{array}\right.
$$


where $\rho, k$ are positive constants.

Let

$$
\begin{aligned}
& V=H_{0}^{1}(\Omega), \quad \quad\|v\|_{V}^{2}=\int_{\Omega}|\nabla u|^{2} d x \\
& H=L^{2}(\Omega), \quad\|v\|_{H}^{2}=\int_{\Omega}|v|^{2} d x
\end{aligned}
$$

and

$$
\mathcal{H}=V \times H \times H, \quad\|(u, v, \theta)\|_{\mathcal{H}}^{2}=\int_{\Omega}\left[|\nabla u|^{2}+|v|^{2}+|\theta|^{2}\right] d x
$$

We then define

$$
D(\mathcal{A})=\{(u, v, \theta) \mid v \in V, \Delta u-\gamma \theta \in H, \gamma v+k \Delta \theta \in H\}
$$

and

$$
\mathcal{A}(u, v, \theta)=(v, \Delta u-\gamma \theta, v+k \Delta \theta) .
$$

This coupled system can be rewritten as an abstract evolution equation on $\mathrm{H}$ :

$$
\left\{\begin{array}{l}
\frac{d}{d t}(u, v, \theta)=\mathcal{A}(u, v, \theta) \\
\left(u(0), v(0), \theta(0)=\left(u_{0}, u_{1}, \theta_{0}\right)\right.
\end{array}\right.
$$

It is known that (see $[\mathrm{KBT}]) e^{t \mathcal{A}}$ strongly stable and is not exponentially stable.

$\mathcal{A}$ satisfies the hypotheses (H1) and (H2). Let us verify (H3) for $l=2$. Suppose it is false, then there exists a sequence $\beta_{n} \in \mathbb{R}^{+}, \beta_{n} \rightarrow \infty$ and $\left(u_{n}, v_{n}, \theta_{n}\right) \in \mathcal{D}(A), n=1,2, \cdots$ with

$$
\left\|\left(u_{n}, v_{n}, \theta_{n}\right)\right\|_{\mathcal{H}}=1, \quad \forall n
$$

and

$$
\beta_{n}^{2}\left(\boldsymbol{i} \beta_{n}-A\right)\left(u_{n}, v_{n}, \theta_{n}\right) \equiv\left(f_{n}, g_{n}, h_{n}\right) \rightarrow 0 \text { in } \mathcal{H}
$$

i.e.,

$$
\begin{aligned}
\beta_{n}^{2}\left(\boldsymbol{i} \beta_{n} u_{n}-v_{n}\right) & =f_{n} \rightarrow 0 \text { in } V, \\
\beta_{n}^{2}\left(\boldsymbol{i} \beta_{n} v_{n}-\Delta u_{n}+\gamma \theta_{n}\right) & =g_{n} \rightarrow 0 \text { in } H, \\
\beta_{n}^{2}\left(\boldsymbol{i} \beta_{n} \theta_{n}-\gamma v_{n}-k \Delta \theta_{n}\right) & =h_{n} \rightarrow 0 \quad \text { in } H .
\end{aligned}
$$

Our goal is to obtain $\left\|\left(u_{n}, v_{n}, \theta_{n}\right)\right\|_{\mathcal{H}} \rightarrow 0$ as $n \rightarrow \infty$, thus a contradiction. 
Since, by (3.21),

$$
-k\left\|\beta_{n} \nabla \theta_{n}\right\|_{H}^{2}=\operatorname{Re}\left\langle\beta_{n}^{2}\left(i \beta_{n}-A\right)\left(u_{n}, v_{n}, \theta_{n}\right),\left(u_{n}, v_{n}, \theta_{n}\right)\right\rangle_{\mathcal{H}} \rightarrow 0
$$

We obtain

$$
\left\|\theta_{n}\right\|_{H} \rightarrow 0
$$

Taking the inner product of (3.25) with $v_{n}$ in $H$ yields

$$
\boldsymbol{i}\left\langle\beta_{n} \theta_{n}, v_{n}\right\rangle_{H}-\left\|v_{n}\right\|_{H}^{2}-\int_{\partial \Omega} v_{n} \frac{\partial \theta_{n}}{\partial \nu} d x+\left\langle\beta_{n} \nabla \theta_{n}, \frac{1}{\beta_{n}} \nabla v_{n}\right\rangle_{H} \rightarrow 0
$$

It follows from (3.23) that $\frac{1}{\beta_{n}} \nabla v_{n}$ is bounded in $H$. Thus the third term in (3.28) converges to zero. The boundary term in (3.28) vanishes due to the boundary conditions. The first term in (3.28) also converges to zero due to (3.26) and the boundedness of $v_{n}$. We have obtained

$$
\left\|v_{n}\right\|_{H} \rightarrow 0
$$

On the other hand, using the sum of the inner product of (3.23) with $v_{n}$ in $H$ and the inner product of (3.24) with $u_{n}$ in $H$,

$$
\left\|u_{n}\right\|_{V}^{2}-\left\|v_{n}\right\|_{H}^{2} \rightarrow 0
$$

Combining (3.27), (3.29) and (3.30), we have the promised contradiction.

Example 3. Consider a Wave equation on a square $\Omega=(0, \pi) \times(0, \pi)$ with local viscous damping

$$
\left\{\begin{array}{l}
u_{t t}(x, t)=\Delta u(x, t)-d(x) u_{t}(x, t) \\
\left.u\right|_{\partial \Omega}=0 \\
u(x, 0)=u_{0}(x), \quad u_{t}(x, 0)=u_{1}(x)
\end{array}\right.
$$

where $d(x)$ has support $\Omega_{0} \subset \Omega$ and is continuous and strictly positive on $\Omega_{0}$, and the damping region $\Omega_{0}$ contains a vertical strip of the square domain, i.e.,

$$
\Omega_{0} \supset \Omega_{s}=\left\{\left(x_{1}, x_{2}\right) \mid a<x_{1}<b, 0<x_{2}<\pi\right\}
$$

Let

$$
\begin{array}{ll}
V=H_{0}^{1}(\Omega), \quad\|v\|_{V}^{2}=\int_{\Omega}|\nabla v|^{2} d x \\
H=L^{2}(\Omega), \quad\|v\|_{H}^{2}=\int_{\Omega}|v|^{2} d x
\end{array}
$$


and

$$
\mathcal{H}=V \times H
$$

Define

$$
\begin{aligned}
& D\left(\mathcal{A}_{d}\right)=\{(u, v) \in \mathcal{H} \mid v \in V, \quad \Delta u \in H\} \\
& \mathcal{A}_{d}(u, v)=(v, \quad \Delta u-d(x) v) .
\end{aligned}
$$

Then the system can be rewritten as an abstract evolution equation on $\mathcal{H}$

$$
\left\{\begin{array}{l}
\frac{d}{d t}(u, v)=\mathcal{A}_{d}(u, v) \\
(u(x, 0), v(x, 0))=\left(u_{0}, u_{1}\right)
\end{array}\right.
$$

We also use $\mathcal{A}_{0}$ to denote $\mathcal{A}_{d}$ when $d \equiv 0$.

It is well known that this system is strongly stable but not exponentially stable since the "geometric optics" condition is violated [BLR]. Clearly, (H1) and (H2) are satisfied. We verify (H3) for $l=2$. Suppose it is false, then there exists $\beta_{n} \in \mathbb{R}^{+}, \beta_{n} \rightarrow \infty$ as $n \rightarrow \infty$ and $z_{n}=\left(u_{n}, v_{n}\right) \in D\left(\mathcal{A}_{d}\right)$ with

$$
\left\|u_{n}\right\|_{V}^{2}+\left\|v_{n}\right\|_{H}^{2}=1
$$

such that

$$
\beta_{n}^{2}\left(\boldsymbol{i} \beta_{n}-\mathcal{A}_{d}\right)\left(u_{n}, v_{n}\right) \rightarrow 0 \quad \text { in } \mathcal{H}
$$

Since

$$
\operatorname{Re}\left\langle\beta_{n}^{2} \mathcal{A}_{d}\left(u_{n}, v_{n}\right),\left(u_{n}, v_{n}\right)\right\rangle_{\mathcal{H}}=\int_{\Omega_{0}} d(x)\left|\beta_{n} v_{n}\right|^{2} d x
$$

we obtain from (3.32) that

$$
\int_{\Omega_{0}} d(x)\left|\beta_{n} v_{n}\right|^{2} d x \rightarrow 0
$$

which further leads to

$$
\lim _{n \rightarrow \infty}\left\|v_{n}\right\|_{L^{2}\left(\Omega_{s}\right)}=0
$$

and

$$
\lim _{t \rightarrow \infty}\left\|\beta_{n}\left(\boldsymbol{i} \beta_{n}-\mathcal{A}_{0}\right) z_{n}\right\|=0 .
$$

It is easy to see that the normalized eigenvectors of $\mathcal{A}_{0}$

$$
\phi_{m, l}(x)=\left(\frac{\sqrt{2}}{\pi \sqrt{m^{2}+l^{2}}} \sin m x_{1} \sin l x_{2}, i \frac{\sqrt{2}}{\pi} \sin m x_{1} \sin l x_{2}\right)
$$


for $m, l=1,2, \cdots$, form an orthonormal basis for $\mathcal{H}$. The corresponding eigenvalue for $\phi_{m, l}(x)$ is

$$
i \lambda_{n}=\boldsymbol{i} \sqrt{m^{2}+l^{2}}
$$

we rearrange the eigenvalues $\left\{\lambda_{k}\right\}$ in the order

$$
\left|\lambda_{1}\right|<\left|\lambda_{2}\right|<\cdots<\left|\lambda_{k}\right|<\cdots
$$

with algebraic multiplicity $i_{k}, 1 \leq i_{k} \leq \operatorname{dim} V_{k}$, where $V_{k}$ is the eigenspace of $\lambda_{k}$. We then expand $z_{n}$ as

$$
z_{n}=\sum_{k, i_{k}}\left\langle z_{n}, \phi_{k}^{i_{k}}\right\rangle \phi_{k}^{i_{k}}
$$

and substitute it into (3.34). This leads to

$$
\lim _{n \rightarrow \infty} \sum_{k, i_{k}} \beta_{n}\left(\boldsymbol{i} \beta_{n}-\boldsymbol{i} \lambda_{k}\right)\left\langle z_{n}, \phi_{k}^{i_{k}}\right\rangle \phi_{k}^{i_{k}}=0,
$$

i.e., for any $\varepsilon>0$, there exists $N_{\varepsilon}>0$ such that

$$
\sum_{k, i_{k}}\left|\beta_{n}\left(\beta_{n}-\lambda_{k}\right)\right|^{2}\left|\left\langle z_{n}, \phi_{k}^{i_{k}}\right\rangle\right|^{2} \leq \varepsilon \quad \text { for all } n \geq N_{\varepsilon}
$$

Lemma 3.1 For each $n$, there exists some index $k(n)$ and a constant $\gamma>0$ so that

$$
\begin{array}{r}
\left|\beta_{n}\left(\beta_{n}-\lambda_{k(n)}\right)\right|^{2}<\varepsilon, \\
\left|\beta_{n}\left(\beta_{n}-\lambda_{k}\right)\right|^{2} \geq \gamma \quad \text { for all } k \neq k(n)
\end{array}
$$

Proof. If (3.40) does not hold, we have $\left|\beta_{n}\left(\beta_{n}-\lambda_{k}\right)\right|^{2} \geq \varepsilon$ for all $k$. This contradicts (3.39) since

$$
\sum_{k, i_{k}}\left|\left\langle z_{n}, \phi_{k}^{i_{k}}\right\rangle\right|^{2}=1
$$

It follows from (3.40) that

$$
\left|\beta_{n}\left(\beta_{n}-\lambda_{k}\right)\right| \geq\left|\beta_{n}\left(\beta_{n}-\lambda_{k(n)}\right)+\beta_{n}\left(\lambda_{k(n)}-\lambda_{k}\right)\right| \geq\left|\beta_{n}\left(\lambda_{k(n)}-\lambda_{k}\right)\right|-\sqrt{\varepsilon}
$$

Since $\beta_{n}$ goes to infinity, (3.41) holds when $\left|\lambda_{k(n)}-\lambda_{k}\right| \geq 1$ for all $k \neq k(n)$. On the other hand, we always have $\left|\lambda_{k(n)}^{2}-\lambda_{k}^{2}\right| \geq 1$ and $\lambda_{k(n)} \geq 1$. Hence, when $\left|\lambda_{k(n)}-\lambda_{k}\right|<1$, we obtain

$$
\begin{aligned}
\left|\beta_{n}\left(\lambda_{k(n)}-\lambda_{k}\right)\right| & =\frac{\left|\beta_{n}\left(\lambda_{k(n)}^{2}-\lambda_{k}^{2}\right)\right|}{\left|\lambda_{k(n)}+\lambda_{k}\right|} \\
& \geq \frac{\left|\beta_{n}\right|}{\left|\lambda_{k(n)}+\lambda_{k}\right|} \\
& \geq \frac{\left|\beta_{n}\right|}{3 \lambda_{k(n)}} \geq \frac{\beta_{n}^{2}}{3\left(\beta_{n}^{2}+\sqrt{\varepsilon}\right)}, \quad \text { by }(3.40) .
\end{aligned}
$$


(3.41) is proved.

Lemma 3.1 and (3.39) implies that

$$
\gamma^{2} \sum_{i_{k}} \sum_{k \neq k(n)}\left|\left\langle z_{n}, \phi_{k}^{i_{k}}\right\rangle\right|^{2}+\left|\beta_{n}\left(\beta_{n}-\lambda_{k(n)}\right)\right|^{2} \sum_{i_{k(n)}}\left|\left\langle z_{n}, \phi_{k(n)}^{i_{k(n)}}\right\rangle\right|^{2} \leq \varepsilon \quad \text { for all } n \geq N_{\varepsilon} .
$$

Define

$$
\psi_{n}=\sum_{i_{k(n)}}\left\langle z_{n}, \phi_{k(n)}^{i_{k(n)}}\right\rangle \phi_{k(n)}^{i_{k(n)}}
$$

Then,

$$
\left\|z_{n}-\psi_{n}\right\|_{\mathcal{H}}^{2}=\sum_{i_{k}} \sum_{k \neq k(n)}\left|\left\langle z_{n}, \phi_{k}^{i_{k}}\right\rangle\right|^{2} \leq \frac{\varepsilon}{\gamma^{2}}
$$

and

$$
\sum_{i_{k(n)}}\left|\left\langle z_{n}, \phi_{k(n)}^{i_{k(n)}}\right\rangle\right|^{2} \geq 1-\frac{\varepsilon}{\gamma^{2}} .
$$

From the second component of $z_{n}-\psi_{n}$,

$$
\left\|v_{n}-i \frac{\sqrt{2}}{\pi} \sum_{i}\left\langle z_{n}, \phi_{k(n)}^{i}\right\rangle \sin m_{i} x_{1} \sin l_{i} x_{2}\right\|_{L^{2}\left(\Omega_{s}\right)} \leq \frac{\varepsilon}{\gamma^{2}}
$$

where

$$
\begin{gathered}
m_{i}^{2}+l_{i}^{2}=m_{k(n)}^{2}+l_{k(n)}^{2}, \quad i=1, \cdots, \operatorname{dim} V_{k(n)} \\
m_{i} \neq m_{j}, \quad l_{i} \neq l_{j} \quad \text { for } \quad i \neq j .
\end{gathered}
$$

On the other hand,

$$
\begin{aligned}
& \frac{2}{\pi^{2}} \int_{a}^{b} \int_{0}^{\pi}\left|\sum_{i}\left\langle z_{n}, \phi_{k(n)}^{i}\right\rangle \sin m_{i} x_{1} \sin l_{i} x_{2}\right|^{2} d x_{2} d x_{1} \\
= & \frac{1}{\pi} \int_{a}^{b} \sum_{i}\left|\left\langle z_{n}, \phi_{k(n)}^{i}\right\rangle\right|^{2} \sin ^{2} m_{i} x_{1} d x_{1} \\
\geq & \delta \sum_{i}\left|\left\langle z_{n}, \phi_{k(n)}^{i}\right\rangle\right|^{2} \\
\geq & \delta\left(1-\frac{\varepsilon}{\gamma^{2}}\right)
\end{aligned}
$$

for some constant $\delta>0$. This contradicts (3.33) and (3.48).

By Theorem 2.1,

$$
\left\|e^{t \mathcal{A}}\left(u_{0}, u_{1}\right)\right\|_{\mathcal{H}} \leq C_{k}\left(\frac{\ln t}{t}\right)^{\frac{k}{2}}(\ln t)\left\|\left(u_{0}, u_{1}\right)\right\|_{\mathcal{D}\left(\mathcal{A}^{k}\right)}
$$




\section{References}

[ALT] K. Ammari, Z. Liu And M. TuCsnak, Decay rates for a beam with pointwise force and moment feedback, Math. Control Signals Systems 15(2002), No. 3, pp. 229-255.

[BLR] C. Bardos, G. LeBeAU AND J. RAUCH, Sharp sufficient conditions for the observation, control and stabilization of waves from the boundary, SIAM J. Cont. Optim., 30(1992), pp. 1024-1065.

[H1] F. L. HuAng, Asymptotic stability theory for linear dynamical systems in Banach spaces, Kexue Tongbao 10 (1983), pp. 584-586. [In Chinese]

[H2] F. L. HuAng, Characteristic condition for exponential stability of linear dynamical systems in Hilbert spaces, Ann. of Diff. Eqs, 1(1) 1985, pp. 43-56.

[H3] F. L. HuAng, Strong asymptotic stability theory for linear dynamical systems in Banach spaces, JDE, Vol. 104, No.2 (1993), pp 307-324.

[JTZ] S. Jaffard, M. Tucsnak And E. Zuazua, Singular inter stabilization of the wave equation, J. Diff. Equa. 145(1998), pp. 184-215.

[KBT $]$ F. A. Khodja, A. Benabdallah and D. Teniou Dynamical stabilizers and coupled systems, ESAIM Proceedings, Vol. 2(1997), pp. 253-262.

[L] BG. LebeAu, Equations des ondes amorties, Algebraic and geometric methods in mathematical physics (Kaciveli, 1993), 73-109, Math. Phys. Stud., 19, Kluwer Acad. Publ., Dordrecht, 1996.

[LL] K. LIU AND Z. LIU, Exponential decay of energy of the Euler-Bernoulli beam with locally distributed Kelvin-Voigt damping, SIAM J. Control Optim., Vol. 36, No. 3 (1998), pp. 1086-1098.

[LbLw] W. LitTman AND B. LiU, On the spectral problems and stabilization of acoustic flow, SIAM J. Appl. Math.59 (1998), pp. 17-34. 
[LY] Z. LIU AND J. YONG, Qualitative properties of certain $C_{0}$ semigroups arising in elastic systems with various dampings, Advances in Differential Equations, Vol. 3, No.5(1998), pp. 643-686.

[LZ] Z. LiU AND S. Zheng, Semigroups associated with dissipative systems, Chapman \& Hall/CRC, 1999.

[Na] R. NAgeL, One-parameter Semigroups of Positive Operators, Lecture Notes in Mathematics, Springer-Verlag, 1980.

[Pr] J. PRüss, On the spectrum of $C_{0}$-semigroups, Trans. Amer. Math. Soc., 284 (1984), pp. $847-857$.

[R] B. RAO, Stabilization of elastic plates with dynamical bounday control, SIAM J. Control Optim., 36 (1998), pp. 148-163.

[RR] J.E.M. RIVERA AND R. RACKE, Polynomial stability in two-dimensional magnetoelasticity, IMA J. Appl. Math. 66(2001), No. 3, pp. 269-283.

[RW] B. RAO AND A. WEHBE, Stabilization frontière de plaques de Kirchhoff avec résolvente non compacte,C.R.Acad.Sci. Paris, 328, Série I (1999), pp. 501-596. 